\title{
BMJ Open Group cognitive behavioural therapy with virtual reality exposure versus group cognitive behavioural therapy with in vivo exposure for social anxiety disorder and agoraphobia: a protocol for a randomised clinical trial
}

\author{
Benjamin Arnfred (D) , ${ }^{1}$ Peter Bang, ${ }^{1,2}$ Carsten Hjorthøj (i) ,1,3 \\ Clas Winding Christensen, ${ }^{1}$ Kirsten Stengaard Moeller, ${ }^{1}$ Morten Hvenegaard, ${ }^{1}$ \\ Lone Agerskov, ${ }^{1}$ Ulrik Krog Gausboel, ${ }^{1}$ Ditte Soe, ${ }^{1}$ Peter Wiborg, ${ }^{1}$ \\ Christopher lan Schøler Smith, ${ }^{1}$ Nicole Rosenberg, ${ }^{1}$ Merete Nordentoft ${ }^{1,4}$
}

To cite: Arnfred B, Bang P, Hjorthøj C, et al. Group cognitive behavioural therapy with virtual reality exposure versus group cognitive behavioural therapy with in vivo exposure for social anxiety disorder and agoraphobia: a protocol for a randomised clinical trial. BMJ Open 2022;12:e051147. doi:10.1136/ bmjopen-2021-051147

- Prepublication history and additional supplemental material for this paper are available online. To view these files, please visit the journal online (http://dx.doi.org/10.1136/ bmjopen-2021-051147).

Received 26 May 2021 Accepted 24 November 2021

Check for updates

(C) Author(s) (or their employer(s)) 2022. Re-use permitted under CC BY-NC. No commercial re-use. See rights and permissions. Published by BMJ.

For numbered affiliations see end of article.

Correspondence to Dr Benjamin Arnfred; barn0006@regionh.dk

\section{ABSTRACT}

Introduction Anxiety disorders have a high lifetime prevalence, early-onset and long duration or chronicity. Exposure therapy is considered one of the most effective elements in cognitive behavioural therapy (CBT) for anxiety, but in vivo exposure can be challenging to access and control, and is sometimes rejected by patients because they consider it too aversive. Virtual reality allows flexible and controlled exposure to challenging situations in an immersive and protected environment.

Aim The SoREAL-trial aims to investigate the effect of group cognitive behavioural therapy (CBT-in vivo) versus group CBT with virtual reality exposure (CBT-in virtuo) for patients diagnosed with social anxiety disorder and/or agoraphobia, in mixed groups.

Methods and analysis The design is an investigatorinitiated randomised, assessor-blinded, parallel-group and superiority-designed clinical trial. Three hundred two patients diagnosed with social anxiety disorder and/ or agoraphobia will be included from the regional mental health centres of Copenhagen and North Sealand and the Northern Region of Denmark. All patients will be offered a manual-based 14-week cognitive behavioural group treatment programme, including eight sessions with exposure therapy. Therapy groups will be centrally randomised with concealed allocation sequence to either CBT-in virtuo or CBT-in vivo. Patients will be assessed at baseline, post-treatment and 1-year follow-up by treatment blinded researchers and research assistants. The primary outcome will be diagnosis-specific symptoms measured with the Liebowitz Social Anxiety Scale for patients with social anxiety disorder and the Mobility Inventory for Agoraphobia for patients with agoraphobia. Secondary outcome measures will include depression symptoms, social functioning and patient satisfaction. Exploratory outcomes will be substance and alcohol use, working alliance and quality of life.

Ethics and dissemination The trial has been approved by the research ethics committee in the Capital Region of Denmark.
Strengths and limitations of this study

The present study will be the first large randomised clinical trial to investigate virtual reality exposure therapy for social anxiety disorder and agoraphobia in group therapy.

- The present study is very closely integrated with clinical practice, making results highly transferable to similar real-life settings.

- Mixing patients with social anxiety disorder and agoraphobia in the same therapy groups have never been investigated systematically, which may confound the interpretation of results.

- Because the study is embedded in an outpatient hospital setting, the intervention was designed to be flexible. This increases the ecological validity but also the risk of systematic bias in treatment administration.

All results, positive, negative as well as inconclusive, will be published as quickly as possible and still in concordance with Danish law on the protection of confidentially and personal information. Results will be presented at national and international scientific conferences. The trial has obtained approval by the Regional Ethics Committee of Zealand $(\mathrm{H}-$ 6-2013-015) and the Danish Data Protection Agency (RHP2014-009-02670). The trial is registered at ClinicalTrial.gov as NCT03845101. The patients will receive information on the trial both verbally and in written form. Written informed consent will be obtained from each patient before inclusion in the trial. The consent form will be scanned and stored in the database system and the physical copy will be destroyed. It is emphasised that participation in the trial is voluntary and that the patient can withdraw his or her consent at any time without consequences for further and continued treatment.

Trial registration number NCT03845101. 


\section{BACKGROUND}

Social anxiety disorder is characterised by paying attention to oneself in an exaggerated manner and having marked fear of being negatively evaluated by other people. ${ }^{12}$ Agoraphobia is characterised by avoidance or enduring with dread, situations in which escape is perceived difficult or where help might not be available in the event of a panic attack, panic-like symptoms or incapacitating symptoms such as loss of bladder and/or bowel control. ${ }^{13}$ Both social anxiety disorder and agoraphobia are associated with marked functional consequences. ${ }^{1}$ In Denmark, anxiety disorders represent the costliest disease burden in terms of lost production, due to their early onset, long duration and high prevalence. ${ }^{4}$

The first-line treatment for social anxiety disorder and agoraphobia is cognitive behavioural therapy (CBT) with exposure therapy. ${ }^{5}$ Several meta-analyses have found that patients with social anxiety disorder and agoraphobia respond well to CBT with exposure therapy, provided in individual as well as group format. ${ }^{7-10}$ Exposure therapy aims to change expectations and emotional responses associated with feared stimuli, by exposing the patient to the stimuli and challenging the patients' expectancies of the likelihood and consequences of a feared outcome. ${ }^{11}$ However, in clinical practice, in-vivo exposure stimuli can be difficult to access and control and patients or therapists sometimes reject the treatment, because they consider it too aversive or too logistically demanding. ${ }^{12-14}$

\section{Virtual reality exposure therapy for social anxiety disorder and} agoraphobia

Virtual reality (VR) technology allows the user to experience virtually mediated environments that are perceived as real or almost real, due to multisensory stimulation and blocking of real-world sensory input. Numerous possibilities for psychological intervention using VR are currently being researched owing to its immersive quality. ${ }^{15}{ }^{16}$ As a therapy tool, VR is most widely used to perform Virtual Reality Exposure Therapy (VRET) ${ }^{16}{ }^{17}$ either as a standalone treatment, for example, ${ }^{18}$ or integrated into a CBT treatment, for example. ${ }^{19}$

The use of VR allows flexible and controlled exposure to challenging situations in an immersive and safe environment. Therefore, using VRET can mitigate the challenges of in-vivo exposure therapy by producing greater user acceptance and access to situations that would otherwise be too difficult to control, too resource-intensive to find and/or have unacceptable confidentiality risks. ${ }^{15} 1920$ Based on this, VRET may improve the efficacy and costeffectiveness of psychotherapeutic interventions for anxiety disorders.

Recent reviews and meta-analysis of VRET, either as a standalone treatment or combined with cognitive interventions, conclude that VRET is more effective than waitlist and placebo control and equally as effective as first-line treatment controls for anxiety disorders. ${ }^{21-23}$ However, in one meta-analysis, the authors find significantly worse treatment effects of VRET for social anxiety disorder, when compared with control groups that received equal amounts of in-vivo exposure. ${ }^{24}$ It has been suggested that it is more difficult to produce VRET environments for social anxiety disorder, as compared with other phobic disorders because human interaction is complex and therefore difficult to realistically recreat ${ }^{25}$ which may explain these results. Accordingly, the same meta-analysis found no significant difference in treatment efficacy for CBT with VRET versus CBT with in-vivo exposure for agoraphobia and specific phobia. ${ }^{24}$

In general, there is a scarcity of high-quality randomised clinical trials evaluating the use of VRET for social anxiety disorder and agoraphobia. ${ }^{16} 2627$ For social anxiety disorder, there are five trials published, the largest having 97 participants. ${ }^{18} 1928-30$ For agoraphobia, there are six trials published, the largest having 80 participants. ${ }^{31-36}$ All in all, the evidence base for using VRET compared with in-vivo exposure for social anxiety disorder and agoraphobia remain small. Therefore, larger studies that capitalise on the unique qualities of VRET are needed.

\section{VR exposure in group therapy}

VRET has never been investigated in a group format. Group therapy for social anxiety disorder and agoraphobia is popular in outpatient settings because it has similar treatment efficacy ${ }^{3-39}$ and is proposed to have better cost efficiency, compared with individual therapy. ${ }^{379}$ However, the claim of cost efficiency for social anxiety disorder is disputed, at least in a UK mental healthcare setting. ${ }^{40}$ Beyond that, therapeutic interpersonal processes such as peer learning and modelling has been suggested to be a distinct benefit of group therapy, ${ }^{41}{ }^{42}$ though this has never been systematically evaluated for mixed anxiety groups. A suggested drawback of group CBT compared with individual CBT is that in-vivo exposure in group therapy is restrained by the logistics of managing several patients simultaneously, leading to comparatively less individualised exposure exercises. ${ }^{43} 44$

The use of VRET in group therapy may therefore be especially beneficial, since it should allow for individualised exposure, as well as a greater amount of exposure therapy because less time will be spent on logistical issues (transport, planning, waiting, and so on), while at the same time retaining the proposed benefits of the therapeutic interpersonal processes and cost-efficiency.

\section{Treatment of social anxiety disorder and agoraphobia in the Danish mental health system}

In the Danish mental health services, patients with social anxiety disorder or agoraphobia as their primary diagnosis are generally offered group CBT. To reduce wait time, patients with these diagnoses are treated in the same therapy groups, generally referred to as 'mixed anxiety groups' or 'phobia groups'. These mixed anxiety groups are considered to be as effective as diagnosisspecific groups, due to the overlap in symptoms and diagnostic criteria, ${ }^{45}$ high degree of comorbidity, ${ }^{46}$ as well as 
recent evidence of the acceptable treatment efficacy of CBT-based transdiagnostic therapies. ${ }^{47}$

However, it is worth noting that the pragmatic mixed anxiety group format has never been systematically evaluated and that the official treatment recommendation remains diagnoses-specific CBT delivered in group or individually. ${ }^{48}$ To maximise the study's clinical representativeness, as defined by Shadish et al, ${ }^{49}$ the treatment structure in the present study, including the comperator, will mimic the treatment offered by the Danish mental health services.

\section{Aim and objectives}

In summary in-vivo exposure is considered effective, but can be challenging to perform. VRET may alleviate these challenges. However, the usefulness of VRET for social anxiety disorder and agoraphobia remains unclear. Larger studies that capitalise on the benefits of VRET are needed. Group therapy may be one way to capitalise on the benefits of VRET because it could allow for more individualised exposure exercises. Mixed anxiety groups are commonly used in Danish mental healthcare to reduce wait time, but have not been systematically evaluated. The treatment, inclusion and exclusion criteria described in the present study match the eligibility criteria for treatment and treatment format of the Danish mental healthcare system to maximise transferability of results to clinical practice.

Therefore, the SoREAL trial aims to evaluate the treatment efficacy of VRET in mixed anxiety CBT groups (CBT-in virtuo) compared with mixed anxiety CBT groups where exposure therapy is performed in-vivo (CBT-in vivo).

Thus, in the SoREAL trial, the following hypotheses' will be tested:

\section{Primary hypothesis}

1. Post-treatment, patients treated with CBT-in virtuo will have a lower level of anxiety symptoms compared with patients treated with CBT-in vivo, measured as total scores on the Liebowitz Social Anxiety Scale (LSAS) for patients with social anxiety disorder and the Mobility Inventory for Agoraphobia (MIA) for patients with agoraphobia converted to the percentage of maximum possible (POMP) scores and averaged within treatment arms.

\section{Secondary hypotheses}

1. One year after treatment, patients treated with CBT-in virtuo will have lower levels of anxiety symptoms compared with patients treated with CBT-in vivo.

2. Post-treatment and 1 year after treatment, patients treated with CBT-in virtuo will have lower levels of fear of negative evaluation compared with patients treated with CBT-in vivo.

Overall, we believe that the SoREAL trial will contribute with knowledge about the efficacy and feasibility of VRE for treating social anxiety disorder and agoraphobia in a clinical outpatient setting. The results of this trial may guide future applications of VR in clinical settings across a wide breadth of use cases.

\section{METHODS AND DESIGN}

This article was written in accordance with the Standard Protocol Items: Recommendations for Interventional Trials (SPIRIT) 2013 explanation and elaboration: guidance for protocols of clinical trials. ${ }^{50}$ The SPIRIT Checklist was followed and the SPIRIT flowchart was used (see online supplemental file 1 and figure 1 ).

\section{Recruitment}

The SoREAL trial is embedded directly into five outpatient clinics offering group CBT for social anxiety disorder and agoraphobia. These clinics are part of the Danish mental healthcare system. To be eligible for treatment in these clinics, patients must be referred by their primary care physicians to a Centre for Visitation and Diagnosis in their area, where their symptomatology will be assessed. At the Centre for Visitation and Diagnosis, they must be referred to one of the five outpatient clinics involved in the study. At the outpatient clinic, the patient will again be clinically assessed, and a diagnosis and treatment plan will be formulated. If social anxiety disorder and/or agoraphobia is considered the primary diagnosis for the patient, they will be asked if they are interested in getting more information about the trial. If they consent to it, their contact details will be given to a researcher, who will invite them to an interview concerning the study.

Mini International Neuropsychiatric Interview (MINI), V. 7.0 for DSM-5 will be used to screen for diagnosis. Psychometric analyses of the MINI have demonstrated acceptable test-retest and inter-rater reliability. ${ }^{51}{ }^{52}$ Diagnostic screening is sufficient due to the thorough assessment from both Centre for Visitation and Diagnostics and the outpatient clinics which must have confirmed social anxiety disorder or agoraphobia as the primary diagnosis of the patient, for the patient to be eligible for the study. If eligibility is confirmed, informed consent is acquired (see online supplemental file 2, for a model consent form). Patients who cannot or will not participate in the study will be offered treatment as usual, which is identical to the control group treatment. Inclusion and exclusion criteria were based on the eligibility criteria for receiving the treatment package in Danish outpatient clinics.

\section{Inclusion criteria}

1. Fulfilling diagnostic criteria for social anxiety disorder and/or agoraphobia.

2. Age $18-75$ years.

3. Sufficient knowledge of the Danish language.

4. Informed consent

Exclusion criteria

1. Alcohol or drug dependence 


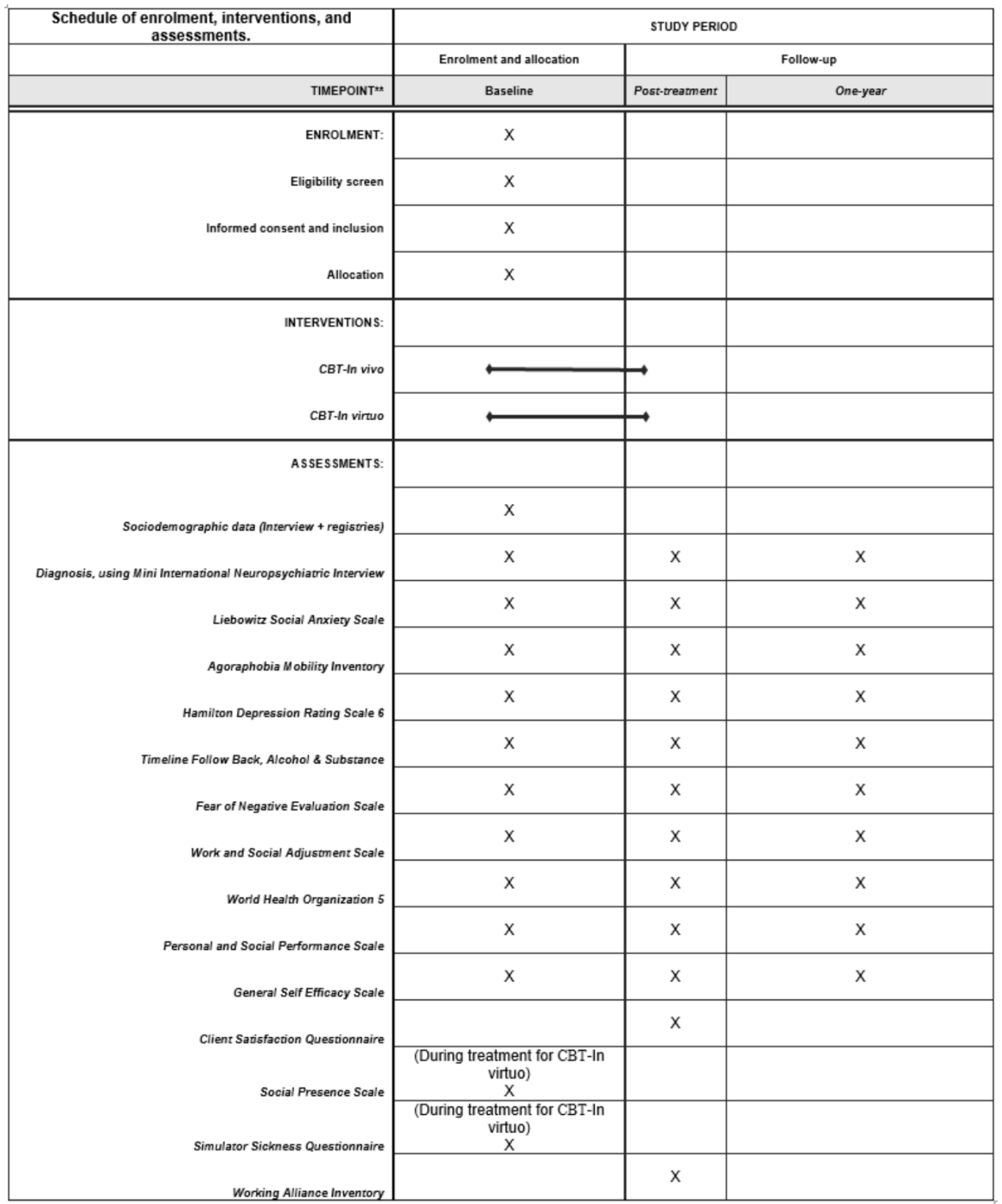

Figure 1 Overview of data collection. CBT, cognitive behavioural therapy.

\section{Feasibility}

Five psychotherapeutic outpatient clinics are involved in the study. All patients referred to these clinics with the relevant diagnosis, who also agree to be contacted, will be invited to an interview about their potential participation. Each of the clinics provide treatment for approximately 30 patients with social anxiety disorder and/or agoraphobia every year. Thus we anticipate that 450 patients will be eligible for the trial during a 3-year recruitment period. We expect a high eligibility rate, due to the previously mentioned assessment procedures the patients will have completed. We also expect a high acceptance rate, due to the novel use of VR technology and the use of a control group that is identical to the treatment they would be offered if they refused participation. See figure 2 for a flow diagram of the SoREAL trial.

\section{Treatment format}

The treatment for social anxiety disorder and agoraphobia offered at the outpatient clinics must follow the national guidelines for the treatment of these disorders. The guidelines are encapsulated in specified 'treatment packages'. For social anxiety disorder and agoraphobia, this package contains:

- 1 hour of assessment.

- 1 hour of individual therapy in preparation for group therapy

- 1 hour of psychometric testing.

- 14 sessions of 2 hours of group therapy

- 1.5 hours of next of kin involvement

- 1 hour of pharmacological treatment planning with a psychiatrist

- 2.5 hours coordination with social services, relapse prevention and follow-up meetings.

Not all of this is necessary for every patient, but every patient can receive every part of the package, should they want to. The treatment in the present study must live up to the standards of the national guidelines. Patients are not allowed to be in any other form of psychotherapeutic treatment.

The therapeutic intervention is manual-based cognitivebehavioural CBT group adapted from the approach of Turk et $a \tilde{l}^{33}$ and Graske and Barlow ${ }^{54}$ with worksheets 


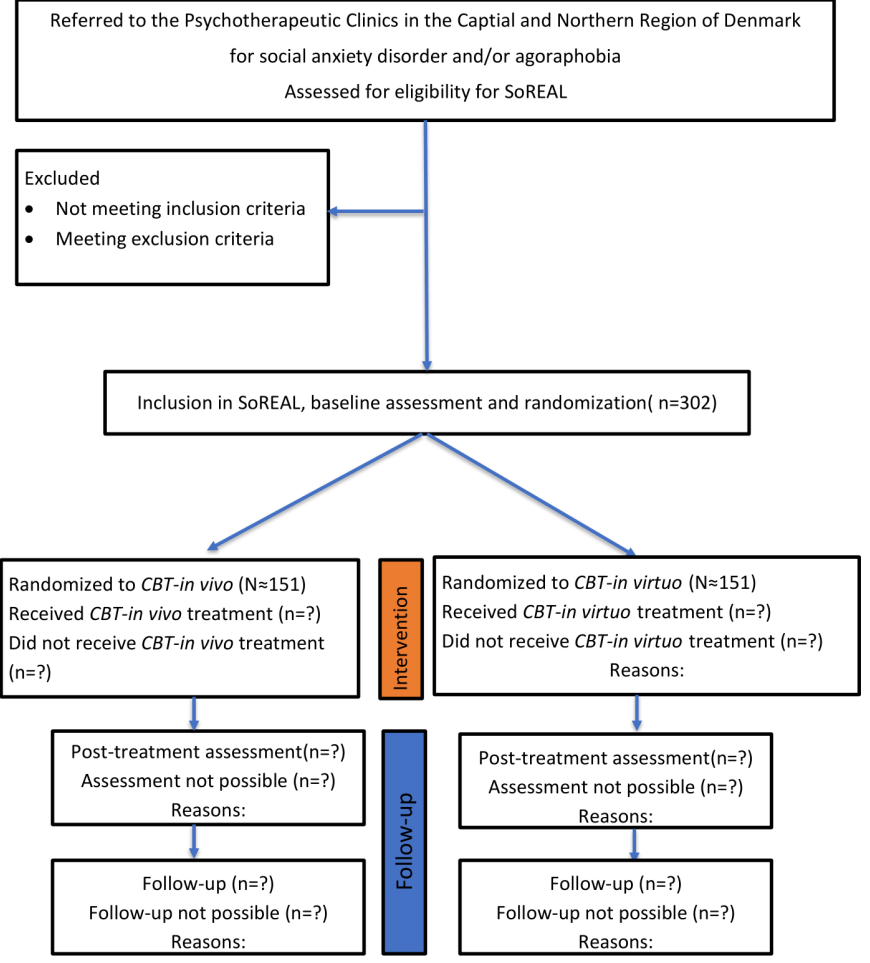

Figure 2 Flow diagram of the SoREAL trial. CBT, cognitive behavioural therapy.

from Rosenberg et $a l^{\tilde{5} 5}$ and inspiration from Bouchard et $a l^{56}$ The treatment will consist of 14 weekly 2-hour group sessions following the manual to ensure equal and uniform treatment for every patient throughout the study. The manual allows flexibility to ensure clinically representative conditions. ${ }^{49}$ For example, it is allowed to change the order of the sessions if it is considered beneficial for the group and multiple exercises are optional. However, the time dedicated to exposure is fixed in both groups. Concurrent psychopharmacological treatment is allowed in both intervention arms.

Groups will consist of 8-9 patients with social anxiety disorder and/or agoraphobia as their primary diagnosis, and every session will be led by two trained clinicians (ie, psychologists, psychiatrists or psychotherapists) with practical experience in CBT and in vivo exposure. Throughout the course of the study, the clinicians involved will treat both CBT-in vivo and CBT-in virtuo groups. Medical consultation, acute individual sessions, supplementary social counselling and physical therapy are possible in both intervention arms. In both intervention arms, the sessions dedicated to exposure are scheduled from the fifth to the eleventh session with approximately $45 \mathrm{~min}$ of exposure in each session. From the fifth session and onwards, all patients in both interventions will have in-vivo exposure as homework. The cognitive therapy strategies used in the non-exposure sessions (first four and last two therapy sessions) are as follows: (1) introduction to CBT; (2) psychoeducation about anxiety and cognitive restructuring of dysfunctional assumptions and beliefs; (3) shifting self-focused attention and modifying
Table 1 Group cognitive behavioural therapy manual session overview for social anxiety disorder and agoraphobia

\begin{tabular}{|c|c|}
\hline Session & Content \\
\hline $\begin{array}{l}\text { Individual } \\
\text { session }\end{array}$ & $\begin{array}{l}\text { Case conceptualisation, psychoeducation on } \\
\text { CBT, treatment goal, introduction to treatment } \\
\text { setting. }\end{array}$ \\
\hline 1 & $\begin{array}{l}\text { Psychoeducation about anxiety, CBT anxiety } \\
\text { model. }\end{array}$ \\
\hline 2 & $\begin{array}{l}\text { Psychoeducation about anxiety, registration of } \\
\text { thoughts, feelings, behaviour and introduction to } \\
\text { cognitive restructuring. }\end{array}$ \\
\hline 3 & $\begin{array}{l}\text { Psychoeducation and exercise: } \\
\text { cognitive bias, attention and self-focus, repetition } \\
\text { about cognitive restructuring, attention exercises. }\end{array}$ \\
\hline 4 & $\begin{array}{l}\text { Psychoeducation about exposure therapy, } \\
\text { optionally, an introductory exposure exercise. }\end{array}$ \\
\hline 5 & Exposure therapy. \\
\hline 6 & Behavioural experiments in exposure exercises. \\
\hline 7 & $\begin{array}{l}\text { Repetition of the methods presented so far, } \\
\text { additional attention/mindfulness exercise linked } \\
\text { to exposure. }\end{array}$ \\
\hline 8 & $\begin{array}{l}\text { Conversational skills and small-talk exposure } \\
\text { exercises. }\end{array}$ \\
\hline 9 & $\begin{array}{l}\text { Introduction to core beliefs, additional exposure } \\
\text { exercises. }\end{array}$ \\
\hline 10 & $\begin{array}{l}\text { Repetition of core beliefs, resources and skills, } \\
\text { additional exposure exercises. }\end{array}$ \\
\hline 11 & Exposure therapy, out of the clinic. \\
\hline 12 & $\begin{array}{l}\text { Repetition and evaluation of methods learnt/used } \\
\text { so far, revising problem-goal list. }\end{array}$ \\
\hline 13 & $\begin{array}{l}\text { Evaluation, discussion and feedback on the } \\
\text { different methods used by each patient. }\end{array}$ \\
\hline 14 & $\begin{array}{l}\text { Maintenance and relapse prevention, review of } \\
\text { skills, review of progress and future goals, plan } \\
\text { for continued exposures, relapse prevention } \\
\text { strategies. }\end{array}$ \\
\hline
\end{tabular}

CBT, cognitive behavioural therapy.

cognitive distortions; (4) developing an understanding of safety behaviour and the rationale of exposure; (5) evaluation, discussion and feedback on the use of patientacquired techniques; and (6) relapse prevention. In both conditions, the exposure exercises aim to develop adaptive responses to anxiety-provoking situations, reinforce cognitive restructuring by framing exercises as behavioural experiments (though these were limited by the non-interactive medium), train attention exercises, train general cognitive strategies (eg, identifying negative automatic thoughts) and train social skills. See tables 1 and 2 for an overview of the content of the CBT sessions for both conditions.

In the in virtuo condition, exposure will take place during 8 out of the 14 group sessions, as in the CBT-in vivo condition. Patients will be exposed to VR situations, 
Table 2 Group CBT manual session overview for social anxiety disorder and agoraphobia with VRET

\begin{tabular}{|c|c|}
\hline Session & Content \\
\hline $\begin{array}{l}\text { Individual } \\
\text { session }\end{array}$ & $\begin{array}{l}\text { Case conceptualisation, psychoeducation on } \\
\text { CBT, treatment goal, introduction to treatment } \\
\text { setting. }\end{array}$ \\
\hline 1 & $\begin{array}{l}\text { Psychoeducation about anxiety, CBT anxiety } \\
\text { model. }\end{array}$ \\
\hline 2 & $\begin{array}{l}\text { Psychoeducation about anxiety, registration of } \\
\text { thoughts, feelings, behaviour and introduction to } \\
\text { cognitive restructuring. }\end{array}$ \\
\hline 3 & $\begin{array}{l}\text { Psychoeducation and exercise: } \\
\text { Cognitive bias, attention and self-focus, } \\
\text { repetition about cognitive restructuring, attention } \\
\text { exercises. }\end{array}$ \\
\hline 4 & $\begin{array}{l}\text { Psychoeducation about exposure therapy, } \\
\text { introduction to VRET. }\end{array}$ \\
\hline 5 & VRET \\
\hline 6 & Behavioural experiments in VRET. \\
\hline 7 & $\begin{array}{l}\text { Repetition of the methods presented so far, } \\
\text { additional attention/mindfulness exercise linked } \\
\text { to VRET. }\end{array}$ \\
\hline 8 & Conversational skills and VRET. \\
\hline 9 & $\begin{array}{l}\text { Introduction to core beliefs, additional VRET } \\
\text { exercises. }\end{array}$ \\
\hline 10 & $\begin{array}{l}\text { Repetition of core beliefs, resources and skills, } \\
\text { additional VRET exercises. }\end{array}$ \\
\hline 11 & $\begin{array}{l}\text { VRET combined with in-vivo out-of-the-clinic } \\
\text { exposure exercises. }\end{array}$ \\
\hline 12 & $\begin{array}{l}\text { Repetition and evaluation of methods learnt/ } \\
\text { used so far, revising problem-goal list. }\end{array}$ \\
\hline 13 & $\begin{array}{l}\text { Evaluation, discussion and feedback on the } \\
\text { different methods used by each patient. }\end{array}$ \\
\hline 14 & $\begin{array}{l}\text { Maintenance and relapse prevention; review of } \\
\text { skills; review of progress and future goals; plan } \\
\text { for continued exposures; relapse prevention } \\
\text { strategies. }\end{array}$ \\
\hline
\end{tabular}

CBT, cognitive behavioural therapy; VRET, virtual reality exposure therapy.

which are relevant to them, and which they are motivated to engage in. Patients in CBT-in virtuo condition will be assigned in vivo exposure homework between sessions in the same way as the CBT-in vivo group.

\section{Fidelity to the treatment manual}

The intervention is manual-based, which improves the standardisation of the treatment. Fidelity to the treatment manual will be assessed through a self-report questionnaire answered by the clinicians at five different time points throughout each group treatment. The questionnaire (and the timepoints when it is delivered) are designed to correspond to the treatment manual. This type of fidelity measurement has proved useful and adequate in trials where the effect of treatment is tested. ${ }^{57}$
The VR headsets will also record statistics of the use of the $360^{\circ}$ films. This data show which specific scenes were watched and how much and can be matched to the individual patient. This data will be used to keep track of the VR usage throughout the study to see how well it matches the treatment manual.

\section{Treatment completion and discontinuation}

Criteria for treatment completion, partial treatment and no treatment were based on clinical guidelines for writing epicrisis as well as discussions within the research group.

- The attendance of 0 or more group therapy sessions will be coded as 'treatment completion'.

- The attendance of between four to nine group therapy sessions will be coded as 'partial treatment'.

- The attendance of less than four group therapy sessions will be coded as 'no treatment'.

Treatment will be discontinued if participants do not show up to treatment 3 weeks in a row and cannot be contacted after multiple attempts by the therapists. Participants who have their treatment discontinued will still be included in the statistical analysis.

\section{VR equipment}

The patients receiving the in virtuo exposure will be immersed using an Oculus Go head-mounted display, enabling viewing of $360^{\circ}$ spherically camera-recorded VR environments. The VR scenarios will thus be highresolution $360^{\circ}$ stereoscopic films, that are played around the viewer. For audio, the patients will use high-quality sound-blocking headphones. For ease of use, the individual videos will be administered from an app that has been designed to be as intuitive to operate as possible. The patient will only have to put on the headset, adjust the focus and choose the desired environment by looking at it in the app. $360^{\circ}$ video was chosen because it gives the most photorealistic visuals, while also being the cheapest to produce. The downside is that it does not allow direct user interaction (eg, the viewer cannot affect the environment in any way). To circumvent this, there are multiple junctions throughout the films where the actors will talk directly and unsolicited to the viewer (eg, greetings, common questions), while also allowing time for the viewer to respond. The actors respond in a generic way to the actions of the viewer. Unsolicited and direct referral from a virtual environment seems to be an essential factor in triggering realistic responses to it. ${ }^{58}$ Though the noninteractability of the environment limits the flexibility of behavioural experiments, it does not make them impossible. For example, it is still possible to hypothesise about internal states (eg, 'I will clam up if I have to present in front of people') and identify and challenge negative automatic thoughts.

\section{VR scenarios}

Thirteen VR exposure scenarios relevant for social anxiety disorder and agoraphobia were chosen for the CBT-in virtuo condition. The 13 scenarios are as follows: 
1. Standing in line in a supermarket.

2. Being in a crowded shopping centre

3. Attending a party.

4. Attending a formal meeting and giving a presentation

5. A job interview.

6. Small talking/discussing in a university canteen with young adults

7. Small talking/discussing in a canteen in a work setting.

8. Entering an auditorium

9. Leaving your apartment

10. Waiting for and taking the bus

11. Crossing a bridge

12. Taking an elevator

13. Taking a commercial aeroplane

Each scenario has four to six scenes of increasing difficulty as well as a neutral scene to familiarise patients with the VR setting. All scenes skip to a looping version of a scene in the same environment after being played, to allow patients to achieve within-session habituation if needed. See online supplemental file 3 for screenshots and descriptions of the individual scenes, as well as links to view a selection of the scenes online. All identifiable persons depicted in the virtual environments are paid actors.

\section{Patient and public involvement: development of VR scenarios and manual}

The pilot phase was a continuous iterative process between the developers of the VR media, CBT-trained clinicians and a panel of patients with social anxiety disorder and/or agoraphobia. The process lasted approximately 16 months (12 for social anxiety disorder environments and 4 for agoraphobia) and consisted of regular meetings following each scenario's initial filming wherein the patients saw the VR scenario in question. Their experience (eg, anxiety level provoked from the films, the validity of the scenarios) was then used as a starting point for a discussion of further development and alterations to the scenarios. Towards the end of the development of the scenarios and application to launch them, two clinicians tested the usability of VRE in a group format. The clinicians and patients then gave further feedback on the films and the delivery of the exposure in the group. This guided the initial draft for a group CBT manual with VRE for social anxiety disorder and agoraphobia.

\section{Assessment}

\section{Diagnostics}

MINI V.7.0 for DSM-5 will be used to screen for diagnosis. At the inclusion interview, all modules but $\mathrm{P}$ will be used to assess diagnostic eligibility. At the baseline interview, all modules but $\mathrm{P}$ will be used to assess diagnosis and detect comorbidity. At the post-treatment interview, all modules but $\mathrm{P}$ will be used to assess diagnosis and detect comorbidity. At the follow-up interview, all modules but $\mathrm{P}$ will be used to assess diagnosis and detect comorbidity.

\section{Outcomes and sample size calculation}

We originally designed the trial around inclusion of only patients with social anxiety disorder, basing the sample size calculation on the following parameters on the LSAS: with alpha $=0.05,80 \%$ power, and an expected SD of 21, 302 patients would be required to detect the minimal relevant difference of 6.8 on the LSAS total score between the groups.

On deciding to expand the diagnostic criteria for inclusion to also include patients with agoraphobia, it was necessary to change our primary outcome measure. For patients with agoraphobia, we primarily rate symptoms using MIA. To include both patients with social anxiety disorder and patients with agoraphobia, we thus decided to recalculate scores on these two scales to POMP as described below. Since the sample size calculation for LSAS was based on a Cohen's $d=0.33$, we also set the minimum clinically relevant difference on MIA, and by extension on the POMP, to $d=0.33$. Consequently, the required sample size remained unaffected by this change of primary outcome measures and is thus still 302 patients. See figure 3 for power calculations on secondary outcomes.

\section{Primary outcome}

Total scores on the LSAS for patients with social anxiety disorder and the MIA for patients with agoraphobia measured pretreatment, post-treatment and at 1-year follow-up converted to the POMP and averaged within treatment arms. POMP calculations can bring differently measured items to the same metric and do not change the multivariate distribution and covariance matrix of the transformed variables. Therefore, scales transformed with the POMP method can be used to examine meanlevel differences between groups. ${ }^{59-61}$ Using POMPtransformed scores on two different measures of phobic anxiety makes it possible to include patients with different primary diagnoses in the same analysis, thus, avoiding the need for approximately double the number of participants to reach a sufficient sample size. The downside of this method is that differences in the sensitivity of the outcome measures and potential differences in treatment effect between patients with social anxiety disorder and agoraphobia, which has been observed in diagnosisspecific treatment, ${ }^{62}$ are also averaged out, thus possibly skewing results.

Social anxiety disorder symptom severity will be measured using a danish version of the LSAS. LSAS assesses 24 situations typically feared by individuals with social anxiety disorder, rated on anxiety and avoidance, divided into subscales of performance anxiety and social situations. It has acceptable psychometric properties. ${ }^{63}$ Agoraphobia symptom severity will be measured using a danish version of the MIA. The MIA assesses avoidance of 26 situations typically feared by patients who were agoraphobic. ${ }^{64}$ The MIA has demonstrated excellent psychometric properties and has been validated in multiple languages, including Swedish. ${ }^{65} 66$ 


\begin{tabular}{|c|c|c|c|c|}
\hline Outcome & $\begin{array}{l}\text { Lowest clinically } \\
\text { relevant difference }\end{array}$ & $\begin{array}{l}\text { Expected } \\
\text { standard } \\
\text { deviation }\end{array}$ & $\begin{array}{l}\text { Calculated } \\
\text { power }\end{array}$ & Reference \\
\hline Fear of Negative Evaluation & 4.5 & 10 & $97 \%$ & {$[68]$} \\
\hline $\begin{array}{l}\text { Hamilton Depression Rating Scale, } \\
6 \text { items }\end{array}$ & 1.6 & 4 & $93 \%$ & [67] \\
\hline Client Satisfaction Questionaire & 2 & 5 & $93 \%$ & [70] \\
\hline WHO Well-Being Index, 5 items & 10 & 25 & $93 \%$ & [71] \\
\hline Work and Social Adjustment Scale & 8 & 10 & $\sim 100 \%$ & {$[69,70]$} \\
\hline Remission $($ LSAS $<30)$ & $\begin{array}{l}20 \% \text { in control group } \\
\text { vs. } \\
35 \% \text { in the VR group }\end{array}$ & & $84 \%$ & {$[63,73]$} \\
\hline $\begin{array}{l}\text { Response (LSAS }<50 \text { or a } 15 \text { point } \\
\text { drop) }\end{array}$ & $\begin{array}{l}65 \% \text { in control group } \\
\text { vs. } 80 \% \text { in VR group }\end{array}$ & & $84 \%$ & [63] \\
\hline Remission (MIA<1.5) & & & & \\
\hline $\begin{array}{l}\text { Response (MIA }<2 \text { or a } 0.5 \text { point } \\
\text { drop) }\end{array}$ & & & & \\
\hline
\end{tabular}

Figure 3 Power calculation for secondary outcomes in the SoREAL trial. LSAS, Liebowitz Social Anxiety Scale; MIA, Mobility Inventory for Agoraphobia; VR, virtual reality.

\section{Secondary outcomes}

- Depressive symptoms measured pretreatment, posttreatment and at follow-up as total scores on the Hamilton Depression Rating Scale, 6 item version (HAM-6) ${ }^{67}$

- Fear of negative evaluation measured pretreatment, post-treatment and at follow-up with the Brief Version of the Fear of Negative Evaluation Scale (FNES). ${ }^{68}$

- Work and social adjustment measured pretreatment, post-treatment and at follow-up with the Work and Social Adjustment Scale (WSAS). ${ }^{69} 70$

- User acceptability and satisfaction of treatment measured post-treatment with the Client Satisfaction Questionnaire (CSQ). The CSQ is an 8-item scale loading to one factor of satisfaction with mental healthcare service. $^{71}$

- Quality of life measured pretreatment, post-treatment and at follow-up with the WHO Well-Being Index, five items (WHO-5). It is considered a very sensitive outcome measure as it does not incorporate negative quality of life, that is, distress, and has no ceiling effect. $^{72}$
- Treatment response on social anxiety disorder symptoms measured as LSAS below 50 or a 15 points drop.

- Treatment response on agoraphobia symptoms measured as MIA below 2 or a 0.5 points drop.

- Remission of social anxiety disorder symptoms measured post-treatment and at follow-up as LSAS below $25^{73}$ and not qualifying for social anxiety disorder as measured using the MINI.

- Remission of agoraphobia symptoms measured posttreatment and at follow-up as MIA below 1.5 and not qualifying for agoraphobia as measured using the MINI.

\section{Explorative outcomes}

- Social functioning measured with Personal and Social Performance Scale ${ }^{74}$ (PSP) pretreatment, posttreatment and at 1-year follow-up.

- Substance and alcohol use measured with timeline followback $^{75}$ (TLFB) pretreatment, post-treatment and at 1-year follow-up.

- Self-belief of coping measured with General Self Efficacy $^{76}$ pretreatment, post-treatment and at 1-year follow-up. 
- Working alliance measured with the Working Alliance Inventory $^{77}$ (WAI) post-treatment.

- Social anxiety symptoms in patients with social anxiety disorder, measured with the LSAS pretreatment, posttreatment and at 1-year follow-up.

- Agoraphobia symptoms in patients with agoraphobia, measured with the MIA pretreatment, post-treatment and at 1-year follow-up.

\section{Other measures}

- Unwanted negative side-effects induced by immersions in VR (commonly referred to as cybersickness) will be measured with the Simulator Sickness Questionnaire $^{78}$ (SSQ) at the end of VRE sessions.

- Deterioration and adverse effects of psychotherapy on social anxiety disorder symptoms measured posttreatment and at follow-up as a $6.8+$ point increase in total LSAS score. Patients who have deteriorated will be interviewed about their experiences in therapy.

- Deterioration and adverse effects of psychotherapy on Agoraphobia symptoms measured post-treatment and at follow-up as a 0.3 point increase in total MIA score. Patients who have deteriorated will be interviewed about their experiences in therapy.

- The experience of social presence, as described by Lee, ${ }^{79}$ will be measured after each VR exposure session with a scale consisting of nine questions rated on a 1-7 Likert scale. This scale was developed specifically for this trial because existing scales are too specific for the VR equipment and content they were developed for. Social presence is measured instead of the more general construct of presence, because it has been theorised to be a critical element in the effective use of VRE for socially related fears. ${ }^{80} 81$

\section{Data from medical report}

The following data will be retrieved from the participants' medical report with consent, only if the participant cannot remember it:

1. Number of previous hospitalisations for mental health conditions or medical conditions.

2. Use of mental health services during the follow-up period

3. Current and previous psychopharmacological medication

4. Attendance rate of the CBT treatment.

\section{Setting of assessment}

Assessment will take place at the outpatient clinics where the patients also receive treatment. Self-report questionnaires (MIA, FNES, CSQ, WAI, WSAS, WHO-5) will be answered by following a link sent to the patient's email address, which the patients can access either on a personal device or on one of the clinic's computers. If preferred by the patient, the self-report questionnaires can be filled out on printed copies of the scales while at the assessment interview. MINI, LSAS, PSP, HAM-D6 and TLFB will be administered by trained researchers and research assistants. After each session with VRE, specific questionnaires (Social Presence \& Simulator Sickness Questionnaire) will be administered by the clinicians delivering the intervention. If necessary, due to the global COVID-19 pandemic, assessment interviews will be performed via telephone.

\section{Randomisation}

Randomisation is performed by randomising each therapy group, 1 week before the first treatment session. This means that no patient is included while their treatment allocation is known. The randomisation is done with a hidden allocation sequence generated from www. sealedenvelope.com and is centralised and handled with the randomisation module in Research Electronic Data Capture (REDCap) by a project manager uninvolved in the data collection. Block sizes will be unknown to the outcome assessors and clinicians. The factor for stratification is the treatment site. Allocation tables will be handled by external researchers with no affiliation with the project. An email of the group's assigned randomisation will be sent to the team leaders organising the logistics of the interventions in the psychotherapeutic clinics. Assigned randomisation of the groups will be stored by the research team data manager. The randomisation code will be stored at REDCap.

\section{Blinding}

The assessors are blinded when interviewing at pretreatment, post-treatment and at follow-up. Should unblinding occur, another researcher will perform the assessment. Blinded researchers will perform analysis and draft conclusions. There are no circumstances where unblinding of the assessors is permissible.

\section{Data collection methods and management}

See figure 1 for an overview of data collection. Selfreported data will be collected through surveys sent via REDCap or filled out on paper. Assessors are trained in the interview instruments and will do regular coratings of recorded interviews. Inter-rater reliability of clinicianrated outcome measures will be calculated throughout the trial. The interviewers will import data from the assessments directly into the electronic Case Report Form using the data entry system REDCap. ${ }^{82}$ REDCap is an electronic data capture tool hosted at Center for IT, Medico and Telephony (CIMT) in the Capital Region of Denmark. For non-self-report measures, data will first be captured on paper and then entered electronically. REDCap complies with Danish legislation (the Act on Processing Personal Data) due to it having both comprehensive user rights and access control management and a complete audit trail on all data transactions. The data from individual patients are tied to a unique serial number. Assigned researchers and Good Clinical Practice (GCP) monitors will be the only people who can access the database. Non-electronic data will be stored locally in secure archives. Data will be exported from REDCap 
without personal identifiers. Data will be exported to all well-known software packages: SPSS v. 28, SAS v. 15.2, Stata v. 17, R v. 4.1.2. and stored on a secure network drive under the control of CIMT. A data manager will ensure that all variables are correctly defined with variable and value labels. All derived variables will be correctly defined, and algorithms will be kept in individual files. All data will be scrutinised to identify errors in data entry. The sponsor and the principal investigators ensure that data are stored at least 10 years after the trial is ended.

\section{Statistical methods}

The analysis will all be from intention-to-treat. All included patients will also be included in the analyses. All statistical tests of significance will be two-tailed. The primary outcome analysis will be an intention-to-treat analysis. Missing data will be handled by multiple imputations $(\mathrm{m}=100)$. As predictors in the imputation model, we will select variables if they are independent predictors of the outcome or predictors of missing data $(p<0.05$ in a univariate model). Each group will have imputations done separately. Analysis of covariance will be used to calculate any significant results between the two groups, using the baseline value and the stratification variables.

The continuous variables will be imputed with linear regression. Binary variables will be imputed with binary logistic regression. Multinomial variables will be imputed with multinomial logistic regression. Ordinal variables will be imputed with ordinal logistic regression. For every type of variable, we will perform 100 imputations.

All distributions will be assessed for normality using visual inspection of histograms and $\mathrm{Q}-\mathrm{Q}$ plots. If not normally distributed, variables will be log-transformed, and if unsuccessful, a non-parametric test will be used.

For dichotomous outcomes, we will perform multiple logistic regressions with treatment as usual as reference and stratification variables as covariates after having imputed missing values using a logistic regression model.

\section{Dissemination}

A trial protocol, including a plan for statistical procedures, has been published at wwwclinicaltrialsgov/ct2/ show/NCT03845101. This will ensure that the SoREAL trial is conducted and analysed as planned. Possible deviations and reasons for those will be described in publications. All data published will be verified for authenticity by controlling for internal inconsistency. All results, positive, negative as well as inconclusive, will be published as quickly as possible and still in concordance with Danish law on the protection of confidentially and personal information. Results will be presented at national and international scientific conferences. Lastly, results will be presented at relevant mental health centres in Denmark.

\section{Data monitoring and auditing}

Like in GCP monitoring, an independent committee will check the following data for the included patients: informed consent, inclusion and exclusion from intervention, serious adverse events and severe adverse reactions. It will be checked whether there is a link between trial allocation and the serious adverse events and severe adverse reactions.

\section{Safety}

In the clinical setting, the clinicians will register adverse events and adverse reactions and report all serious adverse events and severe adverse reactions to the sponsor. Other events or side effects will be collected from patient files and registers. International Conference on Harmonization of Technical Requirements for Registration of Pharmaceuticals for Human Use, Good Clinical Practice guidelines define serious adverse events and serious adverse reactions. The patients in the SoREAL trial are ensured by Danish law and the patient care regulation. Every patient in the SoREAL trial will have access to their results of the trial if they wish to. The clinicians will not have access to data collected from assessments done by the researchers.

\section{Trial status}

Inclusion began on 4 February 2019. Inclusion is expected to stop on 4 June 2023. Inclusion was delayed by approximately 3 months due to the COVID-19 pandemic.

\section{Author affiliations}

${ }^{1}$ Copenhagen Research Center for Mental Health - CORE, Mental Health Center Copenhagen, Copenhagen University Hospital, Copenhagen, Denmark

${ }^{2}$ Department of Biomedical and Clinical Sciences, Linköping University, Linkoping, Sweden

${ }^{3}$ Department of Public Health, University of Copenhagen, Copenhagen, Denmark ${ }^{4}$ Department for Clinical Medicine, Faculty of Health and Medical Science, University of Copenhagen, Copenhagen, Denmark

Contributors Authorship is this based on the Vancouver guidelines. All authors have read, revised and approved the manuscript. MN and NR had the original idea for the trial. MN wrote the application for the NovoNordic Foundation and is the $\mathrm{Pl}$ of the trial. $\mathrm{CH}$ generated the allocation sequence, carried out the power calculations and will be responsible for supervising the statistical analyses. NR was responsible for the non-experimental content of the CBT. CWC, KSM, CISS, PB and BA directed the development of the VR films. CWC, KSM, UKG, DS, PW, BA and PB developed the manual and guidelines for using VRET in group therapy. MH was responsible for outcome measures. BA and PB developed the Social Presence Scale and fidelity measures. BA set up randomisation, built and manage the database and is responsible for all participant assessment, including training and managing research assistants.

Funding MN and NR initiated the project. MN applied to Novo Nordisk Foundation, and the SoREAL trial was granted 5.000.000 DKK [NNF170C0027780]. MN and NR have no affiliation to the Novo Nordisk Foundation. MN, PB and BA applied to TrygFonden and the trial was granted an additional 3.517.500 DKK [ID: 146169]. MN, PB and BA have no affiliation to TrygFonden. The project is entirely independent of the Novo Nordisk Foundation and TrygFonden and therefore, the funding body plays no role in the design of the study, the collection, analysis and interpretation of data and in writing the manuscript. Nor will the Novo Nordisk Foundation or TrygFonden play any role in future publications that may derive from the project.

Competing interests None declared.

Patient consent for publication Not applicable.

Provenance and peer review Not commissioned; externally peer reviewed.

Supplemental material This content has been supplied by the author(s). It has not been vetted by BMJ Publishing Group Limited (BMJ) and may not have been peer-reviewed. Any opinions or recommendations discussed are solely those of the author(s) and are not endorsed by BMJ. BMJ disclaims all liability and 
responsibility arising from any reliance placed on the content. Where the content includes any translated material, BMJ does not warrant the accuracy and reliability of the translations (including but not limited to local regulations, clinical guidelines, terminology, drug names and drug dosages), and is not responsible for any error and/or omissions arising from translation and adaptation or otherwise.

Open access This is an open access article distributed in accordance with the Creative Commons Attribution Non Commercial (CC BY-NC 4.0) license, which permits others to distribute, remix, adapt, build upon this work non-commercially, and license their derivative works on different terms, provided the original work is properly cited, appropriate credit is given, any changes made indicated, and the use is non-commercial. See: http://creativecommons.org/licenses/by-nc/4.0/.

\section{ORCID iDs}

Benjamin Arnfred http://orcid.org/0000-0002-3064-8460

Carsten Hjorthøj http://orcid.org/0000-0002-6943-4785

\section{REFERENCES}

1 American Psychiatric Association. Diagnostic and statistical manual of mental disorders. 5th ed. Washington, DC: American Psychiatric Association, 2013

2 Altwaijri YA, Al-Subaie AS, Al-Habeeb A, et al. Lifetime prevalence and age-of-onset distributions of mental disorders in the Saudi national mental health survey. Int J Methods Psychiatr Res 2020;29:e1836.

3 Craske MG, Barlow DH. Clinical handbook of psychological disorders a step-by-step treatment manual. In: Barlow DH, ed. Clin handb psychol disord a step-by-step treat man. 5th ed. New York, NY, US: The Guilford Press, 2014.

4 Flachs E, Eriksen L, Kock M. Sygdomsbyrden I DANMARK. Copenhagen: Sundhedsstyrelsen, 2015.

5 National Institute for Health and Care Excellence. Social anxiety disorder: assessment and treatment. Natl Inst Heal Care Excell 2013.

6 National Institute for Health and Care Excellence. Generalised anxiety disorder and panic disorder in adults: management. Natl Inst Heal Clin Excell 2011.

7 Barkowski S, Schwartze D, Strauss B, et al. Efficacy of group psychotherapy for social anxiety disorder: a meta-analysis of randomized-controlled trials. J Anxiety Disord 2016;39:44-64.

8 Tolin DF. Is cognitive-behavioral therapy more effective than other therapies? A meta-analytic review. Clin Psychol Rev 2010;30:710-20.

9 Schwartze D, Barkowski S, Strauss B, et al. Efficacy of group psychotherapy for panic disorder: meta-analysis of randomized, controlled trials. Group Dynamics: Theory, Research, and Practice 2017;21:77-93.

10 Pompoli A, Furukawa TA, Efthimiou O, et al. Dismantling cognitivebehaviour therapy for panic disorder: a systematic review and component network meta-analysis. Psychol Med 2018;48:1945-53.

11 O'Donohue W, Fisher JE. Cognitive behavior therapy: core principles for practice. Cogn Behav Ther Core Princ Pract 2012.

12 Neudeck P, Wittchen HU. Exposure therapy: Rethinking the model - Refining the method. In: Neudeck P, Wittchen H, eds. Expo Ther Rethink Model - Refin Method. London: Springer, 2012: 1-358.

13 Butler $\mathrm{G}$. Exposure as a treatment for social phobia: some instructive difficulties. Behav Res Ther 1985;23:651-7.

14 Koch El, Gloster AT, Waller SA. Exposure Treatments for Panic Disorder with and without Agoraphobia. In: Richard DCS, Lauterbach D, eds. Handb Expo ther. Academic Press, 2006: 235-8.

15 Freeman D, Reeve S, Robinson A, et al. Virtual reality in the assessment, understanding, and treatment of mental health disorders. Psychol Med 2017;47:2393-400.

16 Cieślik B, Mazurek J, Rutkowski S, et al. Virtual reality in psychiatric disorders: a systematic review of reviews. Complement Ther Med 2020;52:102480.

17 Maples-Keller JL, Bunnell BE, Kim S-J, et al. The use of virtual reality technology in the treatment of anxiety and other psychiatric disorders. Harv Rev Psychiatry 2017;25:103-13.

18 Kampmann IL, Emmelkamp PMG, Hartanto D, et al. Exposure to virtual social interactions in the treatment of social anxiety disorder: a randomized controlled trial. Behav Res Ther 2016;77:147-56.

19 Bouchard S, Dumoulin S, Robillard G, et al. Virtual reality compared with in vivo exposure in the treatment of social anxiety disorder: a three-arm randomised controlled trial. Br J Psychiatry 2017;210:276-83.

20 Botella C, Fernández-Álvarez J, Guillén V, et al. Recent progress in virtual reality exposure therapy for phobias: a systematic review. Curr Psychiatry Rep 2017;19:42.
21 Carl E, Stein AT, Levihn-Coon A, et al. Virtual reality exposure therapy for anxiety and related disorders: a meta-analysis of randomized controlled trials. J Anxiety Disord 2019;61:27-36.

22 Fodor LA, Cotet CD, Cuijpers P, et al. The effectiveness of virtual reality based interventions for symptoms of anxiety and depression: a meta-analysis. Sci Rep 2018;8:1-13.

23 Chesham RK, Malouff JM, Schutte NS. Meta-Analysis of the efficacy of virtual reality exposure therapy for social anxiety. Behav Change 2018;35:152-66.

24 Wechsler TF, Kümpers F, Mühlberger A. Inferiority or even superiority of virtual reality exposure therapy in phobias? A systematic review and quantitative meta-analysis on randomized controlled trials specifically comparing the efficacy of virtual reality exposure to gold standard in vivo exposure in agoraphobia, specific Phobia, and social Phobia. Front Psychol 2019;10:1758.

25 Emmelkamp PMG, Meyerbröker K, Morina N. Virtual reality therapy in social anxiety disorder. Curr Psychiatry Rep 2020;22:32.

26 Page S, Coxon M. Virtual reality exposure therapy for anxiety disorders: small samples and no controls? Front Psychol 2016;7:1-4.

27 McCann RA, Armstrong CM, Skopp NA, et al. Virtual reality exposure therapy for the treatment of anxiety disorders: an evaluation of research quality. J Anxiety Disord 2014;28:625-31.

28 Anderson PL, Price M, Edwards SM, et al. Virtual reality exposure therapy for social anxiety disorder: a randomized controlled trial. $J$ Consult Clin Psychol 2013;81:751-60.

29 Klinger E, Bouchard S, Légeron P, et al. Virtual reality therapy versus cognitive behavior therapy for social phobia: a preliminary controlled study. Cyberpsychol Behav 2005;8:76-88 http://online.liebertpub. com/doi/abs/

30 Robillard G, Bouchard S, Dumoulin S, et al. Using virtual humans to alleviate social anxiety: preliminary report from a comparative outcome study. Stud Health Technol Inform 2010;154:57-60.

31 Meyerbroeker K, Morina N, Kerkhof GA, et al. Virtual reality exposure therapy does not provide any additional value in agoraphobic patients: a randomized controlled trial. Psychother Psychosom 2013;82:170-6.

32 Botella C, García-Palacios A, Villa H, et al. Virtual reality exposure in the treatment of panic disorder and agoraphobia: a controlled study. Clin Psychol Psychother 2007;14:164-75.

33 Castro WP, Roca Sánchez MJ, Pitti González CT, et al. Cognitivebehavioral treatment and antidepressants combined with virtual reality exposure for patients with chronic agoraphobia. International Journal of Clinical and Health Psychology 2014;14:9-17.

34 Malbos E, Rapee RM, Kavakli M. A controlled study of agoraphobia and the independent effect of virtual reality exposure therapy. Aust $N$ Z J Psychiatry 2013;47:160-8.

35 Malbos E, Rapee RM, Kavakli M. Isolating the effect of virtual reality based exposure therapy for agoraphobia: a comparative trial. Stud Health Technol Inform 2011;167:45-50.

36 Penate W, Pitti CT, Manuel Bethencourt J. The effects of a treatment based on the use of virtual reality exposure and cognitive-behavioral therapy applied to patients with agoraphobia. Int J Clin Heal Psychol 2008;8:5-22.

37 Gould RA, Buckminster S, Pollack MH, et al. Cognitive-behavioral and pharmacological treatment for social phobia: a meta-analysis. Clinical Psychology: Science and Practice 1997;4:291-306.

38 Hans E, Hiller W. A meta-analysis of nonrandomized effectiveness studies on outpatient cognitive behavioral therapy for adult anxiety disorders. Clin Psychol Rev 2013;33:954-64.

39 Roberge P, Marchand A, Reinharz D, et al. Cognitive-behavioral treatment for panic disorder with agoraphobia. Behav Modif 2008;32:333-51.

40 Mavranezouli I, Mayo-Wilson E, Dias S, et al. The cost effectiveness of psychological and pharmacological interventions for social anxiety disorder: a model-based economic analysis. PLoS One 2015;10:e0140704

41 Heimberg RG, Becker R. Cognitive-behavioral group therapy for social phobia: basic mechanisms and clinical strategies. 1st ed. New York: Guilford Press, 2002.

42 Söchting I. Effectiveness of CBGT compared to individual CBT. Cogn Behav Gr Ther 2014:44-58.

43 Hedman E, Mörtberg E, Hesser $\mathrm{H}$, et al. Mediators in psychological treatment of social anxiety disorder: individual cognitive therapy compared to cognitive behavioral group therapy. Behav Res Ther 2013;51:696-705.

44 Mörtberg E, Clark DM, Sundin O, et al. Intensive group cognitive treatment and individual cognitive therapy vs. treatment as usual in social phobia: a randomized controlled trial. Acta Psychiatr Scand 2007:115:142-54. 
45 Meidlinger PC, Hope DA. The new transdiagnostic cognitive behavioral treatments: commentary for clinicians and clinical researchers. J Anxiety Disord 2017;46:101-9.

46 Kessler RC, Chiu WT, Demler O, et al. Prevalence, severity, and comorbidity of 12-month DSM-IV disorders in the National comorbidity survey replication. Arch Gen Psychiatry 2005;62:617-27.

47 Newby JM, McKinnon A, Kuyken W, et al. Systematic review and meta-analysis of transdiagnostic psychological treatments for anxiety and depressive disorders in adulthood. Clin Psychol Rev 2015;40:91-110.

48 Nielsen AC, Zachariassen A, Brendholdt J. KAG Psykoterapi Outcome book 2019-2020 [Internet], 2020. Available: https://www. psykiatri-regionh.dk/undersoegelse-og-behandling/Behandling/ KAGS/KAG-Psykoterapi/Sider/default.aspx

49 Shadish WR, Matt GE, Navarro AM, et al. The effects of psychological therapies under clinically representative conditions: a meta-analysis. Psychol Bull 2000;126:512-29.

50 Chan A-W, Tetzlaff JM, Gøtzsche PC, et al. Spirit 2013 explanation and elaboration: guidance for protocols of clinical trials. BMJ 2013;346:e7586.

51 Sheehan DV, Lecrubier Y, Harnett Sheehan K, et al. The validity of the mini international neuropsychiatric interview (mini) according to the SCID-P and its reliability. European Psychiatry 1997;12:232-41.

52 Lecrubier Y, Sheehan DV, Weiller E, et al. The mini international neuropsychiatric interview (mini). A short diagnostic structured interview: reliability and validity according to the CIDI. Eur Psychiatr. 1997;12:224-31.

53 Turk CL, Heimberg RG, Magee L. Social anxiety disorder. In: Barlow $\mathrm{DH}$, ed. Clin handb psychol disord a step-by-step treat man. London: Guilford Press, 2008: 123-63.

54 Graske MG, Barlow DH. Panic disorder and agoraphobia. In: Barlow $\mathrm{DH}$, ed. Clin handb psychol disord. 4th ed. New York: The Guilford Press, 2008: 1-65.

55 Rosenberg NK, Mørck MM, Arendt M. Kognitiv terapi. Nyeste udvikling. Kogn Ter Nyeste Udvikl 2012

56 Bouchard S, Robillard G, Larouche S. Description of a treatment manual for in virtuo exposure with specific phobia. Virtual Real Psychol Med Pedagog Appl 2012:81-108.

57 Hogue A, Dauber S, Henderson CE, et al. Reliability of therapist selfreport on treatment targets and focus in family-based intervention. Adm Policy Ment Health 2014;41:697-705.

58 Slater M. Place illusion and plausibility can lead to realistic behaviour in immersive virtual environments. Philos Trans $R$ Soc Lond B Biol Sci 2009;364:3549-57.

59 Cohen P, Cohen J, Aiken LS, et al. The problem of units and the circumstance for POMP. Multivariate Behav Res 1999;34:315-46.

60 Fischer R, L. Milfont T. Standardization in psychological research. Int J Psychol Res 2010;3:88-96.

61 Moeller J. A word on standardization in longitudinal studies: don't. Front Psychol 2015;6:1-4.

62 Cuijpers P, Gentili C, Banos RM, et al. Relative effects of cognitive and behavioral therapies on generalized anxiety disorder, social anxiety disorder and panic disorder: a meta-analysis. J Anxiety Disord 2016;43:79-89.

63 Heimberg RG, Horner KJ, Juster HR, et al. Psychometric properties of the Liebowitz social anxiety scale. Psychol Med 1999;29:199-212.
64 Chambless DL, Caputo GC, Jasin SE, et al. The mobility inventory for agoraphobia. Behav Res Ther 1985;23:35-44.

65 Chambless DL, Sharpless BA, Rodriguez D, et al. Psychometric properties of the mobility inventory for agoraphobia: convergent, discriminant, and criterion-related validity. Behav Ther 2011;42:689-99

66 Hoffart A, Øktedalen T, Ulvenes $\mathrm{P}$, et al. The mobility inventory for agoraphobia avoidance alone scale: factor structure and psychometric properties of subscales. Assessment 2018;25:769-76.

67 O'Sullivan RL, Fava M, Agustin C, et al. Sensitivity of the sixitem Hamilton depression rating scale. Acta Psychiatr Scand 1997;95:379-84.

68 Leary MR. A brief version of the fear of negative evaluation scale. Pers Soc Psychol Bull 1983;9:371-5.

69 Zahra D, Qureshi A, Henley W, et al. The work and social adjustment scale: reliability, sensitivity and value. Int J Psychiatry Clin Pract 2014;18:131-8.

70 Mundt JC, Marks IM, Shear MK, et al. The work and social adjustment scale: a simple measure of impairment in functioning. $\mathrm{Br}$ $J$ Psychiatry 2002;180:461-4.

71 Attkisson CC, Zwick R. The client satisfaction questionnaire. Eval Program Plann 1982;5:233-7.

72 Bech $\mathrm{P}$, Olsen LR, Kjoller M, et al. Measuring well-being rather than the absence of distress symptoms: a comparison of the SF-36 mental health subscale and the WHO-five well-being scale. Int $J$ Methods Psychiatr Res 2003;12:85-91.

73 Blanco C, Heimberg RG, Schneier FR, et al. A placebo-controlled trial of phenelzine, cognitive behavioral group therapy, and their combination for social anxiety disorder. Arch Gen Psychiatry 2010;67:286-95.

74 Nasrallah H, Morosini P, Gagnon DD. Reliability, validity and ability to detect change of the personal and social performance scale in patients with stable schizophrenia. Psychiatry Res 2008;161:213-24.

75 Sobell LC, Sobell MB. Timeline follow-back: a technique for assessing self-reported alcohol consumption. Meas alcohol Consum Psychosoc Biochem methods. Totowa, NJ, US: Humana Press, 1992: 41-72.

76 De Las Cuevas C, Peñate W. Validation of the general self-efficacy scale in psychiatric outpatient care. Psicothema 2015;27:410-5.

77 Horvath AO. Working Alliance Inventory - Client form 1992:1-5.

78 Kennedy RS, Lane NE, Berbaum KS. Lilienthal mg. simulator sickness questionnaire: an enhanced method for quantifying simulator sickness. J Comput Inf Technol 2009;5:203-20.

79 Lee KM. Presence, Explicated, 2004: 27-50.

80 Ling Y, Nefs HT, Morina N, et al. A meta-analysis on the relationship between self-reported presence and anxiety in virtual reality exposure therapy for anxiety disorders. PLoS One 2014;9:e96144.

81 Felnhofer A, Hlavacs H, Beutl L, et al. Physical presence, social presence, and anxiety in participants with social anxiety disorder during virtual cue exposure. Cyberpsychol Behav Soc Netw 2019;22:46-50.

82 Harris PA, Taylor R, Thielke R, et al. Research electronic data capture (REDCap)-A metadata-driven methodology and workflow process for providing translational research informatics support. J Biomed Inform 2009:42:377-81. 